Plasma nicht allein die Folge der Ausscheidung, sondern besonders auch der Verteilung im Organismus ist. Dabei gelangt normalerweise ein beträchtlicher Anteil des Aldosterons in die Leber. Fehlt die Leber, so ist der Plasmaschwund erheblich verlangsamt (Abb. 1).

2. bzw. $4 \mathrm{Stdn}$. nach Injektion von $1,2-{ }^{3} \mathrm{H}-\mathrm{d}-A 1 d o-$ steron liegt das freie, d. h. nicht metabolisierte Aldosteron ( $\mathrm{pH}$ 6-Fraktion) im Plasma hepatektomierter Hunde im Mittel in höherer Konzentration vor als bei nicht hepatektomierten Tieren (Tab. 1, Abb. 2).

Die $\mathrm{pH}$ 5-Fraktion, in der das Tetrabydroaldosteronglukuronid enthalten ist, ist dagegen bei den hepatektomierten Tieren im Mittel in etwas geringerer Konzentration im Plasma nachweisbar (Tab. 1, Abb. 2). Dies bestätigt unsere bei den Urinuntersuchungen gemachten Beobachtungen, wonach Aldosteron auch extrahepatisch bis zu einem gewissen Grade in diesen Metaboliten umgewandelt werden kann (1).

Das 3-Oxo-Konjugat ( $\mathrm{pH}$ 1-Fraktion) ist dagegen im Plasma hepatektomierter und nicht hepatektomierter Hunde nur in Spuren vorhanden. Wegen dieser geringen Konzentration lassen Plasmauntersuchungen daher kei- nen eindeutigen Schluß hinsichtlich einer möglichen extrahepatischen Umwandlung von Aldosteron in diesen Metaboliten zu. Unsere im Urin hepatektomierter Hunde durchgeführten Untersuchungen zeigen allerdings deutlich, daß die Metabolisierung des Aldosterons zum 3-Oxo-Konjugat weitgehend extrahepatisch erfolgen kann (1). Die erheblichen Unterschiede in der Konzentration dieses Metaboliten in Plasma und Urin $(1,3)$ sprechen für eine sehr hohe renale Clearance. Beim Menschen konnten wir zeigen, da $\beta$ die Clearance des 3-Oxo-Konjugates bedeutend höher liegt als der renale Plasmadurchfluß, was als strenger Hinweis auf eine in der Niere stattfindende Metabolisierung von Aldosteron zum 3-Oxo-Konjugat aufzufassen ist $(3,4)$. SANDOR und LANTHIER (5) konnten mittels Inkubation von Nierengewebsschnitten mit ${ }^{14} \mathrm{C}$-d-Aldosteron zeigen, $\mathrm{da} \beta$ in der Niere eine Konjugation von Aldosteron in die Glukuronidfraktion einerseits und in die säurelabile Fraktion (3-Oxo-Konjugat) andererseits möglich ist. Weitere Untersuchungen sind notwendig, um Auskunft über das Ausmaß dieser Metabolisierung in der Niere zu erhalten.

\title{
Literatur
}

1. ManN, M., W. Siegenthaler; K. Krampr und E. ZingG, Klin. Wschr. 42, 319 (1964). - 2. Ulick, S., J. biol. Chemistry 236, 680 (1961). - 3. Siegenthaler, W., R. Peterson und G. Frimpter, in: An International Symposium on Aldosterone, Blackwell Scientific Publications Ltd., Oxford, im Druck (1964). -
4. Gfeller, J., Die renale Clearance von freiem Aldosteron vor und nach Applikation eines Aldosteronantagonisten. Inaug. Diss. Zürich (1964). - 5. SANDor, T. und A. Lanthier, Acta endocr., K'hvn. 39, 87 (1962).

\section{pH-Abhängigkeit der Wirkung von Butazolidin und Tanderil}

\author{
Von \\ H. Holzer und B. Ulrich \\ Aus dem Biochemischen Institut der Universität Freiburg im Breisgau (Direktor: Prof. Dr. H. Holzer) \\ (Der Schriftleitung zugegangen am 6. April 1964)

\begin{abstract}
„Tanderil““ (1-Phenyl-2-(p-hydroxyphenyl)-3,5-dioxo-4-n-butyl-pyrazolidin) hemmt ebenso wie „Butazolidin“ (1,2Diphenyl-3,5-dioxo-4-n-butyl-pyrazolidin) in schwach saurem Milieu die Glykolyse von Ascites-Zellen um ein Vielfaches stärker als im neutralen Milieu. Da entzündete Gewebe und ihre Umgebung schwach sauer sind, könnte die Spezifität der Entzündungs-hemmenden Wirkung von Butażolidin und Tanderil mit den pH-abhängigen Permeationseigenschaften zusammenhängen.

„Tanderil“ (1-phenyl-2-(p-hydroxyphenyl)-3,5-dioxo-4-n-butyl-pyrazolidine) and „butazolidine“ (1,2-diphenyl-3,5dioxo-4-n-butyl-pyrazolidine) inhibit glycolysis in ascites cells far more. strongly in a weakly acidic medium than in a neutral medium. Since the inflamed tissues are weakly acidic, the specificity of the anti-inflammatory action of butazolidine and tanderil may be due to their $\mathrm{pH}$-dependent permeation properties.
\end{abstract}

Wie wir in früheren Versuchen fanden (1), ist die Hemmung der Atmung und Glykose von EhrlichAscites-Zellen durch „Butazolidin“ (1,2-Diphenyl-3,5dioxo-4-n-butyl-pyrazolidin) vom $\mathrm{pH}$ abhängig. Bei $\mathrm{pH}=6$ findet man eine mehr als 10 fach stärkere Glykolysehemmung als bei $\mathrm{pH}=7,2$. $\mathrm{Da}$ entzündete Gewebe ein saureres $\mathrm{pH}$ aufweisen als Normalgewebe, ist es möglich, daß die stärkere Wirksamkeit des Butazolidin in saurerem Milieu an der Spezifität der Entzündungshemmung beteiligt ist. Sehr wahrscheinlich ist es zwar nicht eine Hemmung der Atmung oder der Glykolyse, die die Entzündungshemmung bewirkt, jedoch können 
diese Hemmeffekte als $\mathrm{Maß}$ für die pH-Abhängigkeit der Wirkung von Butazolidin benützt werden. - Nicht nur Butazolidin selbst, sondern auch sein Metabolit "Tanderil"“ (1-Phenyl-2-(p-hydroxyphenyl)-3,5-dioxo-4n-butyl-pyrazolidin) haben Entzündungs-hemmende Wirkung. In der vorliegenden Arbeit wird die Frage geprüft, ob auch Tanderil die charakteristische $\mathrm{pH}$ Abhängigkeit der Wirkung zeigt.

\section{Tab. 1}

Glykolysehemmung bei $\mathrm{pH}=6,0 \mathrm{bzw} . \mathrm{pH}=7,2$

Pro Gefäß $370 \mu l$ Ehrlich-Ascites-Zellen (Gewinnung vgl. (2) und (3)) in Krebs-Ringer-Bicarbonat-Lösung mit 0,8\% Glucose. Gesamtvolumen $3,0 \mathrm{ml}$. Für $\mathrm{pH}=6,0$ Gasphase: $100 \% \mathrm{CO}_{2}$, für $\mathrm{pH}=7,2$ Gasphase: $95 \% \mathrm{~N}_{2}+5 \% \mathrm{CO}_{2}$ (vgl. (1)). In der Tabelle sind $\mu l \mathrm{CO}_{2}$ pro Gefäß pro Stunde angegeben. In Klammern: Prozent der Kontrolle ohne Zusatz

\begin{tabular}{lccccc}
\hline \multicolumn{1}{c}{ Zusatz } & - & Tanderil & \multicolumn{2}{c}{ Butazolidin } \\
\hline $\begin{array}{l}\text { Konzentration } \\
(\mathrm{mg} \%=\mathrm{mg} / 100 \mathrm{~m} l)\end{array}$ & - & 13,3 & 3,3 & 3,3 & 0,8 \\
\hline $\mathrm{pH}=6,0$ & 112 & 39 & 85 & 25 & 48 \\
& $(100 \%)$ & $(35 \%)$ & $(76 \%)$ & $(22 \%)$ & $(43 \%)$ \\
\hline $\mathrm{pH}=7,2$ & 448 & 535 & 516 & 518 & 420 \\
& $(100 \%)$ & $(119 \%)$ & $(115 \%)$ & $(116 \%)$ & $(94 \%)$ \\
\hline
\end{tabular}

Wie man aus Tabelle 1 sieht, finden wir bei $\mathrm{pH}=6,0$ mit 3,3 bzw. 13,3 mg\% Tanderil Hemmung der anaeroben Glykolyse von Ascites-Zellen auf 76 bzw. 35\% der Kontrollen, während bei $\mathrm{pH}=7,2$ keine Hemmung zu beobachten ist. $\mathrm{Ob}$ die geringe bei $\mathrm{pH} 7,2$ regelmäßig von uns beobachtete Aktivierung signifikant ist, haben wir nicht weiter geprüft. Im Vergleich zu unseren früheren Versuchen mit Butazolidin (1) beobachteten wir jetzt mit einer etwa $10 \mathrm{mal}$ geringeren Konzentration Glykolysehemmung. Da die verwendeten Tumorstämme verschieden waren, dürfte die Ursache hierfür in verschiedener Empfindlichkeit verschiedener Stämme bestehen.
Tab. 2

DPN-Gehalt von Ascites-Zellen nach Glykolysehemmung durch Tanderil und Butazolidin

Pro Gefäß $252 \mu l$ Zellen. $\mathrm{pH}=6,0$; sonst wie bei Tab. 1. NSA = Nicotinsäureamid (Endkonzentration 0,02 M). Tanderil und Butazolidin je $13 \mathrm{mg}$ pro $100 \mathrm{ml}$. Zahlenangaben: $\mu l \mathrm{CO}_{2}$ pro Gefä pro Stunde (in Klammern: \% der Ansätze ohne Tanderil bzw. Butazolidin); $\mu \mathrm{Mol}$ DPN pro Gefäß nach Abstoppen mit $\mathrm{HClO}_{4}$ (Endkonzentration 5\%), Zentrifugieren, Neutralisieren des Überstandes mit $\mathrm{KHCO}_{3}, 20 \mathrm{Min}$. stehen lassen bei $0^{\circ}$, Zentrifugieren und Analyse des Überstandes im optischen Test nach (4)

\begin{tabular}{lccccccc}
\hline & Kontrollen & \multicolumn{2}{c}{ Tanderil } & \multicolumn{2}{c}{ Butazolidin } \\
\hline & - & NSA & - & NSA & - & NSA \\
\hline$\mu l \mathrm{CO}_{2}$ & 113 & 126 & $46(41 \%)$ & $34(27 \%)$ & $6(5 \%)$ & $3(2 \%)$ \\
\hline$\mu \mathrm{M}$ DPN & 0,014 & 0,022 & 0,015 & 0,025 & 0,012 & 0,024 \\
\hline
\end{tabular}

Zur Frage des Mechanismus der Glykolysehemmung haben wir geprüft, ob die DPN-Konzentration verändert wird. Tabelle 2 zeigt, daß dies auch bei fast vollständiger Glykolysehemmung nicht der Fall ist. $\mathrm{Da}$ die den DPN-Gehalt erhöhende Wirkung von Nicotinsäureamid durch Butazolidin und Tanderil nicht beeinflußt wird, kann eine Beeinflussung des Kohlenhydratstoffwechsels durch Wechselwirkung mit DPN oder Eingriff in den DPN-Stoffwechsel ausgeschlossen werden. - Unsere Versuche geben keine Auskunft über den Entzündungs-hemmenden Mechanismus der Wirkung von Tanderil bzw. Butazolidin. Sie ermöglichen jedoch eine Erklärung für den spezifischen Effekt dieser Substanzen auf entzündete Gewebe. Wahrscheinlich ist es das bei saurem Milieu beschleunigte Eindringen in Zellen, das bei gleichmäßiger Verteilung im Organismus zu der selektiven Wirkung in den entzündeten Geweben mit ihrer saureren Umgebung beiträgt. Da auch Tumoren saurer als Normalgewebe sind, kann man eine spezifische Einwirkung auch auf diese Gewebe erwarten. Untersuchungen hierüber sind uns nicht bekannt.

\section{Literatur}

1. Sedparayr, G., A. Kemnitz und H. Holzer, Klin. Woschr. 34, 1114 (1956). - 2. Holzer, H., J. HaAN und D. Pette, Biochem. Z. 327, 195 (1955). - 3. Holzer, H., G. Sedlmayr und A. Kem-
NITZ, Biochem. Z. 328, 163 (1956). - 4. HolzER, H., S. GoldSCHMid, W. LAMprecht und E. HeLmReich, Hoppe-Seyler's Z. Physiol. Chem. 297, 1 (1954).

Professor Dr. rer. nat. Helmut Holzer Biochemisches Institut der Universität 78 Freiburg im Breisgau Hermann-Herder-Straße 7

\section{BUCHBESPRECHUNGEN}

Handbuch der Protoplasmaforschung Bd. II/D1 : Vitalfärbung und Vitalfluorochromierung tierischer Zellen. Von L. STOCKINGER. IV, 96 S., 15 Abb. (davon 1 farbige Tafel), Gr. -8 ${ }^{\circ}$, DM 31,一. Springer-Verlag, Wien 1964.

Dieses Buch will keinen umfassenden Ưberblick über die physikochemischen Grundlagen der Vitalfärbung geben. Der Verfasser baut bewußt auf den klassischen Darstellungen von ZEIGER (1938) und RIEs (1938) auf. Er hat die notwendige Aufgabe übernommen, das seither erarbeitete Material zusammenzufassen und zu deuten.
Zum besseren Verständnis sind aber die Grundlagen verständlich und sehr einprägsam - oft in Tabellenform - kurz erläutert. Dadurch wird dieses Buch zu einem praktischen Nachschlagewerk auch für Untersucher aus anderen, nicht rein morphologischen Fachgebieten. Darüber hinaus liegt der Wert dieses Buches in der Verarbeitung von Befunden, die in neuerer Zeit durch andere Methoden (Biochemie, Elektronenmikroskopie, Autoradiographie, Histochemie) erhoben wurden. Biochemisch konnten neue Zellfraktionen und funktionell wichtige Substanzen isoliert und ihre Bindung an Vitalfarbstoffe nachgewiesen werden. Die Elek- 